\title{
Enfermagem e imunização aos pacientes imunodeprimidos relacionados à neoplasia
}

\section{maligna}

\author{
Nursing and immunization for immunodepressed patients related to malignant neoplasia \\ Enfermería e inmunización para pacientes inmunodeprimidos relacionados con neoplasia maligna
}

Recebido: 26/12/2020 | Revisado: 26/12/2020 | Aceito: 30/12/2020 | Publicado: 04/01/2021

Mary Hellem Silva Fonseca

ORCID: https://orcid.org/0000-0003-2797-916X Universidade do Estado do Rio de Janeiro, Brasil

E-mail: maryhellem96@gmail.com

Mercedes Neto

ORCID: https://orcid.org/0000-0001-7529-9535 Universidade do Estado do Rio de Janeiro, Brasil

E-mail: mercedesneto.uerj@gmail.com

Eliza Aguiar de Almeida

ORCID: https://orcid.org/ 0000-0003-1551-9502 Universidade do Estado do Rio de Janeiro, Brasil

E-mail: elizaa.aguiar@gmail.com

Ricardo de Mattos Russo Rafael

ORCID: https://orcid.org/0000-0003-1315-4271

Universidade do Estado do Rio de Janeiro, Brasil

E-mail: prof.ricardomattos@gmail.com

Sonia Acioli de Oliveira

ORCID: https://orcid.org/ 0000-0002-0772-8235

Universidade do Estado do Rio de Janeiro, Brasil

E-mail: soacioli@gmail.com

Fernando Porto

ORCID: https://orcid.org/0000-0002-2880-724X

Universidade Federal do Estado do Rio de Janeiro, Brasil

E-mail: ramosporto@openlink.com.br

\begin{abstract}
Resumo
Introdução: A imunização é uma medida de prevenção e controle para determinadas doenças, e em condições normais, as células do corpo humano são capazes de responder a inúmeros estímulos infecciosos ou antigénicos, além da vacinação. Objetivo: descrever os aspectos particulares da imunização em pacientes em tratamento oncológico. Metodologia: Estudo do tipo revisão integrativa. A análise foi realizada após aplicação dos critérios de inclusão e exclusão, e construído um banco de dados contemplando os itens título, ano de publicação, periódico, qualis CAPES, delineamento do estudo, cenários e país. Resultados: Foram 24 artigos analisados, 58,3\% sem classificação de qualis, e nos demais, frequência de qualis em A2 (60,0\%). O delineamento do estudo concentrou 54,1\% no nível 02 de evidência científica. As vacinas descritas nos estudos foram em um total de 11, com destaque para a Influenza com ocorrência de 83,3\%. Para a soroconversão, o resultado positivo apresentou-se em 31,9\% (15/47). Considerações Finais: Conclui-se que as produções são concentradas em alto nível de evidência científica, denotando experimentos para aplicação de vacinas atenuadas em pacientes imunocomprometidos por neoplasia maligna.
\end{abstract}

Palavras-chave: Imunização; Neoplasia; Quimioterapia; Enfermagem; Saúde pública.

\begin{abstract}
Introduction: Immunization is a measure of prevention and control for certain diseases, and under normal conditions, the cells of the human body are able to respond to numerous infectious or antigenic stimuli, in addition to vaccination. Objective: to describe the particular aspects of immunization in patients undergoing cancer treatment. Methodology: Integrative review study. The analysis was carried out after applying the inclusion and exclusion criteria, and a database was built covering the items title, year of publication, periodical, CAPES qualis, study design, scenarios and country. Results: 24 articles were analyzed, $58.3 \%$ without qualification classification, and in the others, frequency of qualifications in A2 (60.0\%). The study design concentrated $54.1 \%$ at level 02 of scientific evidence. The vaccines described in the studies were a total of 11 , with emphasis on Influenza with an occurrence of $83.3 \%$. For seroconversion, the positive result was $31.9 \%$ (15/47). Final Considerations: It is concluded that the productions are concentrated in a high level of scientific evidence, denoting experiments for the application of attenuated vaccines in patients immunocompromised by malignant neoplasms.
\end{abstract}

Keywords: Immunization; Neoplasia; Chemotherapy; Nursing; Public health. 


\begin{abstract}
Resumen
Introducción: La inmunización es una medida de prevención y control de determinadas enfermedades y, en condiciones normales, las células del cuerpo humano son capaces de responder a numerosos estímulos infecciosos o antigénicos, además de la vacunación. Objetivo: describir los aspectos particulares de la inmunización en pacientes sometidos a tratamiento oncológico. Metodología: Estudio de revisión integradora. El análisis se realizó luego de aplicar los criterios de inclusión y exclusión, y se construyó una base de datos con el título de los ítems, año de publicación, revista, calificación CAPES, diseño del estudio, escenarios y país. Resultados: se analizaron 24 artículos, 58,3\% sin clasificación de calificación, y en los demás, frecuencia de calificaciones en A2 (60,0\%). El diseño del estudio concentró el 54,1\% en el nivel 02 de evidencia científica. Las vacunas descritas en los estudios fueron un total de 11, con énfasis en Influenza con una ocurrencia del $83,3 \%$. Para la seroconversión, el resultado positivo fue del 31,9\% (15/47). Consideraciones finales: Se concluye que las producciones se concentran en un alto nivel de evidencia científica, denotando experimentos para la aplicación de vacunas atenuadas en pacientes inmunodeprimidos por neoplasias malignas.
\end{abstract}

Palabras clave: Inmunización; Neoplasia; Quimioterapia; Enfermería; Salud pública.

\title{
1. Introdução
}

A imunização é uma medida de prevenção e controle para determinadas doenças, proporcionando à prevenção de riscos e agravos a população. Esta ação pode ser proporcionada por meio de imunização ativa ou passiva (Brasil, 2014).

A imunização passiva é indicada para indivíduos, não imunes, que se tornam expostos a uma doença infecciosa, e a imunização ativa não está disponível, está contraindicada ou ainda não tenha sido administrada antes da exposição. Para esta imunidade passiva, soros e imunoglobulinas são administrados (Tavares et al., 2005). Na imunização ativa, além de se adquirir pela doença, a outra forma é a vacinação, que é realizada por meio da administração do imunobiológico que vai confeccionar ao indivíduo a proteção imunológica de uma doença infecciosa específica (Brasil, 2014).

O Programa Nacional de Imunizações (PNI) fundamenta-se no controle, erradicação ou eliminação de doenças imunopreveníveis. Criado em 1973, o seu conhecimento é de nível internacional por manter qualidade em suas ações e conquistas, como a erradicação da poliomielite, a eliminação da circulação do vírus autóctone da rubéola, além da varíola, e outras tantas doenças imunopreveníveis que já não mais circulam em território nacional (Silva Junior, 2013).

Em condições normais, as células do corpo humano são capazes de responder a inúmeros estímulos infecciosos ou antigénicos recebidos por um tipo de agravo a saúde, ou mesmo à vacinação. Um paciente neoplásico tem a alteração desse evento, ou seja, como consequencia sua imunidade fica comprometida perdendo algumas de suas defesas adquiridas, pelo fato de existir uma doença e pelo tratamento imunossupressor, o que resulta em uma resposta imune reduzida após a vacinação. Esta população se denomina imunocomprometidos ou imunodeprimidos (Brasil, 2014).

O Centro de Referência para Imunobiológicos Especiais (CRIE) são unidades que atendem grupos de pacientes que se encaixam na categoria de imunização a indivíduos em situações especiais necessitando de imunobiológico específico, e dentre eles se encontram os imunocomprometidos ou imunodeprimidos, por exemplo os com neoplasia maligna (Brasil, 2014).

O risco de um paciente com câncer desenvolver infecções é alto, podendo ocasionar em hospitalização, interrupção da quimioterapia, pneumonias ou até a morte. Um das infecções de maior ocorrência nestes pacientes é por influenza, o que configura incidência de 03 a 05 vezes maior do que na população geral. Assim, a imunização nestes pacientes tem como objetivo fornecer proteção contra infecções imunopreveníveis, diminuindo a quantidade de pacientes vulneráveis (Sanada et al., 2016).

A imunização de indivíduos imunodeprimidos devido ao câncer ou a imunodepressão terapêutica, deve ser levado em conta não só o paciente neoplásico, como também as pessoas de sua convivência. Por isso que a vacinação nesse caso tem que ser avaliada em um contexto amplo, incluindo o médico oncologista do paciente e os profissionais do CRIE, que irão avaliar o tratamento imunossupressor balizado a vacinação (Brasil, 2014).

Durante o período de tratamento quimioterápico, o paciente imunodeprimido passa por duas fases. Na fase mais intensa, 
a vacinação mais eficaz são contra Hepatite A e B. Na fase menos intensa, as vacinações com toxóide, subunidades proteicas ou antígenos bacterianos são mais efetivas. Além da varicela, a administração de vacinas de vírus vivos atenuados não é recomendada durante esta fase (Cesaro et al., 2014).

Após o tratamento, os níveis séricos de anticorpos se tornam insuficientes, e a recomendação é que haja avaliação do caso, e orientação para renovação de vacinas antes aplicadas, sejam elas em esquema completo ou doses de reforço. Uma vez que a recuperação imunológica é completada, geralmente após 6 meses, a resposta ao reforço ou vacinação é geralmente boa e permite que os pacientes sejam protegidos e também contribuir para a imunidade de rebanho (Cesaro et al., 2014).

Este cuidado de vacinação deste público se dá por meio de avaliação de cada caso, pelo oncologista e pelo enfermeiro da sala de vacinas, localde atuação no processo saúde e doença, oferecendo prevenção de doenças imunopreveníveis aos usuários, sejam eles vacinas de rotina, ou aqueles em situações especiais encaminhados ao serviço de imunização (Queiroz et al., 2009).

Sendo assim, a equipe de enfermagem tem total importância na prestação do cuidado em imunização dos pacientes oncológicos, sendo tanto por administrar a vacina, como agente orientador e promotor de conhecimento sobre os imunobiológicos que são específicos para pacientes neoplásicos (Queiroz et al., 2009 \& Oliveira et al., 2010).

Desse modo, visando à imunização de pacientes neoplásicos em situação de imunodepressão, este estudo tem como objeto a imunização de pacientes neoplásicos em tratamento quimioterápico. Para operacionalizar a pesquisa, traçou-se o objetivo: descrever os aspectos particulares da imunização em pacientes em tratamento oncológico.

\section{Metodologia}

Trata-se de uma revisão integrativa de literatura que consiste em um método com etapas de pesquisa pré-definidas rigorosamente. A revisão integrativa proporciona uma síntese de conhecimento e a incorporação da aplicabilidade de resultados de estudos significativos na prática (Souza et al., 2009).

A revisão integrativa contém um processo de elaboração constituído por seis etapas que nortearam esse trabalho, a saber: identificação do tema e da questão de pesquisa; estabelecimento dos critérios de inclusão e exclusão; identificação dos estudos pré-selecionados e selecionados; categorização dos estudos selecionados; análise e interpretação dos resultados e apresentação da revisão/síntese do conhecimento (Souza et al., 2009).

Com o intuito de guiar a pesquisa, foi formulada a seguinte questão de pesquisa: Quais vacinas são aplicadas e testadas em pacientes oncológicos e quais taxas de soroconversão? A seleção dos artigos foi realizada no período de março a abril de 2018, por meio do portal regional da BVS.

Através de consultas no DeCS, foram extraídos os descritores em português e seus respectivos sinônimos submetidos a pesquisa para identificação de seus entretermos. O processo de busca foi direcionado pelos descritores neoplasia, imunização e vacinas. Para o descritor Neoplasia, os sinônimos foram Câncer/tumor/neoplasias/tumores/tumores malignos/neoplasia benigna/neoplasia maligna/ malignidade/cancro (tumor maligno). Para o descritor Imunização foram utilizados Estimulação imunológica/imunoestimulação/sensibilização imunológica/variolação/imunizações, e para Vacinas, Vacina.

No momento do cruzamento feito entre os descritores e os sinônimos acima, foi utilizada a lógica booleana com o uso dos operadores AND e OR. Todos os descritores e seus respectivos sinônimos foram cruzados com um total de 04 cruzamentos. Foram adotados como critério de inclusão artigos disponíveis na integra indexados nas bases de dados LILACS e MEDLINE, publicados nos últimos 05 anos, nos idiomas português e inglês. Foram excluídos artigos que não respondiam a pergunta de pesquisa e artigos duplicados (Stillwell et al., 2010).

O cruzamento foi feito com os descritores e seus respectivos sinônimos. Para o primeiro cruzamento, neoplasia OR (sinônimos) AND imunização OR (sinônimos) AND vacinas OR (sinônimos), totalizou 1.603 artigos encontrados. Para o 
segundo cruzamento, neoplasia OR (sinônimos) AND imunização OR (sinônimos), totalizou 2.758 artigos. O terceiro cruzamento foi feito pelos descritores neoplasia OR (sinônimos) AND vacinas OR (sinônimos), totalizando 5.329 artigos. Por fim, o quarto cruzamento, imunização OR (sinônimos) AND vacinas OR (sinônimos), totalizando 5.188 artigos.

Após a leitura dos títulos e resumos, os estudos selecionados foram analisados buscando identificar se os mesmos contemplavam a questão norteadora deste estudo. O processo de amostragem resultou em um total de 24 artigos.

A fim de viabilizar as publicações selecionadas para a análise, foi realizado um banco de dados elaborado pela pesquisadora, contemplando itens como: título, ano de publicação, periódico, qualis CAPES, delineamento do estudo, cenários e país.

\section{Resultados e Discussão}

As publicações sob a temática de imunização em pacientes neoplásicos, segundo esta pesquisa, apresentaram descobertas em seu perfil, mas também nos resultados de orientações sobre a vacinação a este grupo, e potencial efetividade desta ação de prevenção.

\subsection{Perfil das publicações sobre imunização em pacientes Oncológicos}

Nesta seção, o objetivo foi descrever o perfil das publicações encontradas na busca realizada na BVS sobre imunização em pacientes oncológicos. Foram encontrados 24 artigos, que foram organizados no Quadro 1, onde cada artigo recebeu um número arábico para que fosse mais bem identificado no estudo.

Quadro 1. Distribuição dos artigos selecionados para a revisão integrativa. Rio de Janeiro. Brasil, 2018.

\begin{tabular}{|l|l|l|l|l|}
\hline $\mathbf{N}^{\mathbf{0}}$ & Título & Ano & Periódico & $\begin{array}{l}\text { Qualis CAPES } \\
\text { Enfermagem }\end{array}$ \\
\hline 1 & $\begin{array}{l}\text { Protection against vaccine preventable diseases in children } \\
\text { treated for acute lymphoblastic leukemia. }\end{array}$ & 2016 & $\begin{array}{l}\text { Pediatric Blood \& } \\
\text { Cancer }\end{array}$ & - \\
\hline 2 & $\begin{array}{l}\text { Immunogenicity and safety of high-dose trivalent inactivated } \\
\text { influenza vaccine compared to standard-dose vaccine in } \\
\text { children and young adults with cancer or HIV infection }\end{array}$ & 2016 & Vaccine & A2 \\
\hline 3 & $\begin{array}{l}\text { Cellular and humoral influenza-specific immune response upon } \\
\text { vaccination in patients with common variable } \\
\text { immunodeficiency and unclassified antibody deficiency }\end{array}$ & 2016 & Vaccine & A2 \\
\hline 4 & $\begin{array}{l}\text { A prospective study on the efficacy of two-dose influenza } \\
\text { vaccinations in cancer patients receiving chemotherapy }\end{array}$ & 2016 & $\begin{array}{l}\text { Japanese Journal of } \\
\text { Clinical Oncology }\end{array}$ & - \\
\hline 5 & $\begin{array}{l}\text { Vaccination of chemotherapy patients-effect of guideline } \\
\text { implementation }\end{array}$ & 2016 & $\begin{array}{l}\text { Supportive Care in } \\
\text { Cancer }\end{array}$ & A2 \\
\hline 6 & $\begin{array}{l}\text { A Population-Based, Nationwide Cross-Sectional Study on } \\
\text { Influenza Vaccination Status among Cancer Survivors in Korea }\end{array}$ & 2015 & $\begin{array}{l}\text { Revista Internacional } \\
\text { de Pesquisa } \\
\text { Ambiental e Saúde } \\
\text { Pública }\end{array}$ & B1 \\
\hline 7 & $\begin{array}{l}\text { Failure of a Single Varicella Vaccination to Protect Children } \\
\text { with Cancer From Life-Threatening Breakthrough Varicella }\end{array}$ & 2015 & $\begin{array}{l}\text { The Pediatric } \\
\text { Infectious Disease } \\
\text { Journal }\end{array}$ & - \\
\hline 8 & $\begin{array}{l}\text { Quality Improvement Initiative to Increase Influenza } \\
\text { Vaccination in Pediatric Cancer Patients }\end{array}$ & 2015 & $\begin{array}{l}\text { American Academy } \\
\text { of Pediatrics }\end{array}$ & - \\
\hline 9 & $\begin{array}{l}\text { Influenza Vaccination and Associated Factors among Korean } \\
\text { Cancer Survivors: A Cross-Sectional Analysis of the Fourth \& } \\
\text { Fifth Korea National Health and Nutrition Examination } \\
\text { Surveys }\end{array}$ & 2014 & $\begin{array}{l}\text { Journal Korean } \\
\text { Medicine Science }\end{array}$ & - \\
\hline
\end{tabular}




\begin{tabular}{|c|c|c|c|c|}
\hline 10 & $\begin{array}{l}\text { Influenza Vaccination Coverage Among Adult Survivors of } \\
\text { Pediatric Cancer }\end{array}$ & 2014 & $\begin{array}{l}\text { American journal of } \\
\text { Preventive Medicine }\end{array}$ & - \\
\hline 11 & $\begin{array}{l}\text { Guidelines on Vaccinations in PaediatricHaematology and } \\
\text { Oncology Patients }\end{array}$ & 2014 & $\begin{array}{l}\text { BioMed Research } \\
\text { International }\end{array}$ & A2 \\
\hline 12 & $\begin{array}{l}\text { Vaccination for seasonal influenza in patients with cancer: } \\
\text { recommendations of the Italian Society of Medical Oncology } \\
\text { (AIOM) }\end{array}$ & 2014 & Annals of Oncology & - \\
\hline 13 & $\begin{array}{l}\text { Factors associated with influenza vaccination among adult } \\
\text { cancer patients: a case-control study }\end{array}$ & 2014 & $\begin{array}{l}\text { Clinical Microbiology } \\
\text { and Infection Journal }\end{array}$ & - \\
\hline 14 & $\begin{array}{l}\text { Influenza vaccination in children being treated with } \\
\text { chemotherapy for cancer }\end{array}$ & 2013 & $\begin{array}{l}\text { Cochrane Database } \\
\text { Syst Rev. }\end{array}$ & - \\
\hline 15 & Protecting Pediatric Oncology Patients from Influenza & 2013 & The Oncologist & - \\
\hline 16 & $\begin{array}{l}\text { Influenza vaccination among cancer survivors: disparities in } \\
\text { prevalence between blacks and whites }\end{array}$ & 2013 & $\begin{array}{l}\text { Journal of Cancer } \\
\text { Survivorship }\end{array}$ & - \\
\hline 17 & $\begin{array}{l}\text { Influenza Vaccination in Patients With Cancer: Factors } \\
\text { Associated With Vaccination Practices for Patients and Their } \\
\text { Household Members }\end{array}$ & 2015 & $\begin{array}{l}\text { Infection Control \& } \\
\text { Hospital } \\
\text { Epidemiology }\end{array}$ & - \\
\hline 18 & $\begin{array}{l}\text { Influenza vaccination in adult patients with solid tumours } \\
\text { treated with chemotherapy }\end{array}$ & 2017 & $\begin{array}{l}\text { European Journal of } \\
\text { Cancer }\end{array}$ & A1 \\
\hline 19 & $\begin{array}{l}\text { Varicella vaccination in pediatric oncology patients without } \\
\text { interruption of chemotherapy. }\end{array}$ & 2016 & $\begin{array}{l}\text { Journal of Clinical } \\
\text { Virology }\end{array}$ & A2 \\
\hline 20 & $\begin{array}{l}\text { Improved immunogenicity of high-dose influenza vaccine } \\
\text { compared to standard-dose influenza vaccine in adult oncology } \\
\text { patients younger than } 65 \text { years receiving chemotherapy: A pilot } \\
\text { randomized clinical trial }\end{array}$ & 2016 & Vaccine & $\mathrm{A} 2$ \\
\hline 21 & $\begin{array}{l}\text { Hepatitis B Virus Seroprevalence Among Children with } \\
\text { Cancer in Sudan }\end{array}$ & 2015 & $\begin{array}{l}\text { Pediatric Blood \& } \\
\text { Cancer }\end{array}$ & - \\
\hline 22 & $\begin{array}{l}\text { Immunogenicity and clinical effectiveness of the trivalent } \\
\text { inactivated influenza vaccine in immunocompromised children } \\
\text { undergoing treatment for cancer }\end{array}$ & 2016 & Cancer Medicine & - \\
\hline 23 & Influenza Vaccines in Immunosuppressed Adults with Cancer & 2014 & $\begin{array}{l}\text { Clinical Journal of } \\
\text { Oncology Nursing }\end{array}$ & A1 \\
\hline 24 & $\begin{array}{l}\text { Immunization practices in acute lymphocytic leukemia and } \\
\text { post-hematopoietic stem cell transplant in Canadian Pediatric } \\
\text { Hematology/Oncology centers }\end{array}$ & 2016 & $\begin{array}{l}\text { Human Vaccines \& } \\
\text { Immunotherapeutics }\end{array}$ & B1 \\
\hline
\end{tabular}

Fonte: Autores.

Mediante os dados coletados e apresentados no Quadro 1, as categorias elencadas foram ano, periódico e qualis. Os artigos encontrados, segundo critérios de inclusão no estudo, compreenderam entre os anos de 2013 e 2017, com 12,5\% (03) em 2013; 25,0\% (06) em 2014, 20,8\% (05) em 2015, 37,5\% (09) em 2016 e 4,2\% (01) em 2017.Isso pode indicar que este tema teve crescente publicação durante o decorrer desses anos e, portanto, acredita-se que surgiram interesses para temática entre os pesquisadores de saúde. No entanto, em 2017com percentual de 4,2\% de publicação, leva a inferir que, ou a direção da publicação dos artigos sobre o tema ainda não foram aceitos para publicação ou o se tornou de menor importância para os profissionais de saúde.

Com relação aos periódicos, se faz evidente o destaque das produções serem em revistas específicas da temática, principalmente - Vacinação e Oncologia. Foram 16,7\% (04) publicações em revistas sob o tema Vacina, e 37,5\% (09) em revistas de Oncologia. O destaque dos resultados nesta pesquisa se dá na única publicação na área da Enfermagem - Clinical Journal of Oncology Nursing, o que pode indicar possível tema emergente nesta área de conhecimento (Lisy, 2014).

O item qualis apresentado no Quadro 1, foi preenchido por meio de classificação realizada na plataforma Sucupira do portal de identificação "Qualis Capes” no CNPq. As revistas foram registradas no campo "título”, e marcando o item "área de 
avaliação" com Enfermagem. O objetivo foi verificar a repercussão e representação destes periódicos nesta área de conhecimento. Dos 24 artigos, a 58,3\% (14 artigos) não apresentaram classificação qualis. Dos 41,7\% (10 artigos),20\% (02) foram classificados como A1; 60\% (06) classificados como A2, e 20\% (02), classificados com qualis B1. No universo de 10 publicações com qualis, 80,0\% apresentaram qualis na estratificação A (A1 ou A2), o que configura que este tema tem publicação em periódicos relevantes. Ademais, há de se destacar que a única revista de enfermagem nos resultados desta pesquisa com publicação obteve qualis A1 na Enfermagem, corroborando com as demais avaliações das revistas.

Outro aspecto observado sobre as publicações com a temática imunização em pacientes neoplásicos pesquisadas neste estudo foram o país e cenário de desenvolvimento da pesquisa, bem como o nível de evidência. Destaca-se que, para o nível de evidência, os artigos foram classificados considerando um sistema de classificação composto por sete níveis: 1 - revisões sistemáticas ou metanálise de relevantes ensaios clínicos; 2 - evidências de pelo menos um ensaio clínico randomizado controlado bem delineado; 3 - ensaios clínicos bem delineados sem randomização; 4 - estudos de coorte e de caso-controle bem delineados; 5 - revisão sistemática de estudos descritivos e qualitativos; 6 - evidências derivadas de um único estudo descritivo ou qualitativo; 7 - opinião de autoridades ou comitês de especialistas incluindo interpretações de informações não baseadas em pesquisas (Souza et al., 2009).

Quadro 2. Distribuição dos artigos por cenário e evidência de estudo. Rio de Janeiro, Brasil, 2018.

\begin{tabular}{|l|l|l|l|}
\hline $\mathbf{N}^{\mathbf{0}}$ & Delineamento do estudo/ nível de evidência & Cenário & País \\
\hline 1 & Ensaio clínico randomizado. & Saint-Justine Pediatric Care Center & Canadá \\
\hline 2 & Ensaio clínico randomizado. & St. Jude Children's Research Hospital (SJCRH) & EUA \\
\hline 3 & Ensaio clínico sem randomização & $\begin{array}{l}\text { Institute of Medical Immunology at the Charité } \\
\text { Universitätsmedizin Berlin }\end{array}$ & EUA \\
\hline 4 & Ensaio clínico sem randomização & $\begin{array}{l}\text { Kobe University Hospital and Hyogo Prefectural AWAJI } \\
\text { Medical } \\
\text { Centre }\end{array}$ & Japão \\
\hline 5 & Ensaio clínico sem randomização & St. Jude Children's Research Hospital (SJCRH) & EUA \\
\hline 6 & Ensaio clínico sem randomização & Korea National Health and Nutrition Examination Survey & Korea \\
\hline 7 & Ensaio clínico randomizado. & $\begin{array}{l}\text { Albany Medical Center, no Departamento de Saúde e no } \\
\text { Hospital Infantil da Universidade de Iowa }\end{array}$ & EUA \\
\hline 8 & Ensaio clínico sem randomização & $\begin{array}{l}\text { oncology inpatient unit and } \\
\text { outpatient clinic sites at the } \\
\text { Children's Hospital of Philadelphia } \\
\text { (CHOP) }\end{array}$ & EUA \\
\hline 12 & Transversal & Korea National Health and Nutrition Examination & Korea \\
\hline 10 & Transversal & $\begin{array}{l}\text { Sistema de Vigilância de Fatores de Risco } \\
\text { Comportamental de 2009 }\end{array}$ & EUA \\
\hline 11 & Validade & $\begin{array}{l}\text { Associação Italiana de Oncologia Pediátrica de } \\
\text { Hematologia (AIEOP) }\end{array}$ & Itália \\
\hline & Sociedade Italiana de Virologia e da Autoridade Sanitária & Itália \\
\hline
\end{tabular}


Research, Society and Development, v. 10, n. 1, e10210111516, 2021

(CC BY 4.0) | ISSN 2525-3409 | DOI: http://dx.doi.org/10.33448/rsd-v10i1.11516

\begin{tabular}{|l|l|l|l|}
\hline & & Italiana & \\
\hline 13 & Caso-controle & $\begin{array}{l}\text { prontuários dos pacientes e validados através de } \\
\text { entrevistas pessoais }\end{array}$ & Israel \\
\hline 14 & Revisão sistemática & $\begin{array}{l}\text { (CENTRAL), MEDLINE, EMBASE, (ICAAC), (IDSA)r } \\
\text { (MASCC) (SIOP) }\end{array}$ & EUA \\
\hline 15 & Revisão sistemática & PubMed, Cochrane & EUA \\
\hline 16 & Ecológico & $\begin{array}{l}\text { Sistema de Vigilância de Fator de Risco Comportamental } \\
\text { de 2009 }\end{array}$ & EUA \\
\hline 17 & Ensaio clínico sem randomização & Laboratório Universitário & EUA \\
\hline 18 & Ensaio clínico sem randomização & Institute for Public Health and the Environment & Holanda \\
\hline 19 & Coorte & Academic Medical Center & Holanda \\
\hline 20 & Ensaio clínico sem randomização & Rochester General Hospital (RGH) em Rochester, NY & EUA \\
\hline 21 & Ensaio clínico randomizado & Radioisotope Center in Khartoum & Sudão \\
\hline 22 & Ensaio clínico randomizado & $\begin{array}{l}\text { Departamento de Hematologia Clínica e Oncologia, } \\
\text { Hospital Princesa Margaret para Crianças (PMH }\end{array}$ & Austrália \\
\hline 23 & Revisão Sistemática & $\begin{array}{l}\text { CENTRAL. MEDLINE, LILACS. ICAAC, ECCMID, } \\
\text { IDSA, ASH, ASBMT, EBMT e ASCO }\end{array}$ & EUA \\
\hline 24 & Descritivo Qualitativa & $\begin{array}{l}\text { Canadian Pediatric Hematology/ } \\
\text { Oncology centers. }\end{array}$ & Canadá \\
\hline
\end{tabular}

Fonte: Autores.

No Quadro 2 verifica-se que, dentre os 24 artigos, nenhum artigo foi classificado como nível de evidência 1 ou 7. Dentre os classificados, 20,8\% (05) foram classificados como nível de evidência 2, 33,3\% (08) são classificados como nível de evidência 3, 8,4\% (02) são classificados como nível de evidência 4, 16,7\% (04) são classificados como nível de evidência 5, 20,8\% (05) são classificados como nível de evidência 6. Destarte, as publicações neste quesito de análise também corroboram com a qualidade dos produtos científicos, entendendo que $54,1 \%$ dos artigos foram classificados com nível de evidência alto para as publicações

No entanto, apesar do artigo publicado na revista da área da Enfermagem ter apresentado qualis de qualidade, na categoria de nível de evidência ficou classificado como5, que pode ser inferido pela baixa publicação sobre a temática, ou pela metodologia aplicada, ou mesmo pelo parco investimento em pesquisas sobre imunização pela área.

Foram identificados também os países nos quais as pesquisas foram realizadas, e eles classificados segundo o índice de desenvolvimento humano, o potencial de desenvolvimento e subdesenvolvimento destas nações. Foram 91,7\% (22) de cenários em países desenvolvidos, 4,2\% (01) em países em desenvolvimento, e 4,2\% (01) em países subdesenvolvidos o que se direciona a inferência de pesquisas de ponta em sua qualidade balizada ao investimento em ciência próprio dos países desenvolvidos.

Sendo assim, entende-se que as publicações sobre imunização de pacientes neoplásicos são produtos de pesquisas desenvolvidas em países desenvolvidos, com potente investimento em produção de ciência e conhecimento, com evidência alta e concentrada em produtos da área da Medicina, onde a Enfermagem é quase que imperceptível em sua representação, mesmo sendo temática de sua prática nas salas de vacina e consultórios de imunização. 


\subsection{Vacinas e taxas de soroconversão em pacientes neoplásicos imunizados}

Apesar de entender que a imunização é uma ação de prevenção de agravos, uma prática estabelecida nas salas de vacinas e exercida pela equipe de enfermagem, com publicações por pesquisadores fora desta área, há de se compreender a efetividade destas vacinas aos pacientes neoplásicos.

Quadro 3. Resultados de Pesquisa dos Artigos da Revisão Integrativa, Brasil, 2018.

\begin{tabular}{|c|c|c|c|}
\hline $\mathbf{N}^{\circ}$ & Vacina & Indicações & $\begin{array}{l}\text { Soroconversão sem } \\
\text { interrupção da QT }\end{array}$ \\
\hline \multirow{3}{*}{1} & $\begin{array}{l}\text { Tétano, difteria, poliomielite } 3 \text { e } \\
\text { caxumba }\end{array}$ & $\begin{array}{l}\text { Após a quimioterapia, menos de } 50 \% \text { dos } \\
\text { pacientes foram soroprotegidos }\end{array}$ & Não \\
\hline & TT, DT, polio1, sarampo e rubéola & $\begin{array}{l}\text { Após a quimioterapia a taxa de proteção } \\
\text { aumentou } 90 \% \text { após a segunda dose }\end{array}$ & Não \\
\hline & varicela-zóster e pólio 3 & Permaneceu abaixo dos limiares de proteção & Não \\
\hline 2 & Influenza - trivalente inativada & $\begin{array}{l}\text { Dose padrão: } 40 \% \text { após a dose } 1 \text { e } 20 \% \text { após a } \\
\text { dose } 2 \text { de soroconversão } \\
\text { Alta dose: } 54 \% \text { após a dose } 1 \text { e } 38 \% \text { após a dose } \\
2 \text { de soroconversão }\end{array}$ & Sim \\
\hline 3 & Influenza sazonal sem adjuvante & $\begin{array}{l}\text { Tiveram frequências similares de células T } \\
\text { produtoras de IFN- } \gamma \text {, TNF- } \alpha \text { e IL-2 CD40L (+) } \\
\text { como o grupo controle }\end{array}$ & Sim \\
\hline 4 & Influenza - Duas doses & $\begin{array}{l}\text { Na segunda dose a proporção daqueles com } \\
\text { títulos de proteção contra as cepas individuais } \\
\text { aumentou em } 10 \% \text { (H1N1), } 8 \%(\mathrm{H} 3 \mathrm{~N} 2) \text { e } 3 \% \\
\text { (B) em comparação com a primeira dose }\end{array}$ & Sim \\
\hline \multirow{3}{*}{5} & Pneumocócica conjugada & $\begin{array}{l}\text { Indicada para antes do início da quimioterapia ou } \\
\text { depois do tratamento }\end{array}$ & Não \\
\hline & Influenza - trivalente inativada & $\begin{array}{l}\text { Indicada ao paciente oncológico (indicado duas } \\
\text { doses para uma melhor taxa) }\end{array}$ & Sim \\
\hline & Varicela (VZV) & $\begin{array}{l}\text { Contra indicada durante a QT. Indicada antes de } \\
\text { começar o tratamento }\end{array}$ & Não \\
\hline 6 & Influenza & - & - \\
\hline 7 & Varicela & $\begin{array}{l}\text { Uma criança com apenas uma dose administrada } \\
\text { antes do tratamento quimioterápico, tem a } \\
\text { mesma classificação que um imunossuprimido } \\
\text { não imunizado. }\end{array}$ & Não \\
\hline 8 & Influenza & - & - \\
\hline 9 & Influenza & - & - \\
\hline 10 & Influenza & - & - \\
\hline 11 & Influenza & - & Sim \\
\hline
\end{tabular}




\begin{tabular}{|c|c|c|c|}
\hline & Sarampo e caxumba & $\begin{array}{l}\text { Suspender quimioterapia para uma melhor taxa } \\
\text { de soroconversão }\end{array}$ & Não \\
\hline & Varicela $(\mathrm{VZV})$ & Contra indicado durante a quimioterapia & Não \\
\hline & $\begin{array}{l}\text { Poliomielite, coqueluche, tétano, } \\
\text { difteria, hepatite, Pneumococo e } \\
\text { meningococo }\end{array}$ & $\begin{array}{l}\text { Resposta de anticorpos potencialmente sub- } \\
\text { ótima, resultando em eficácia reduzida em } \\
\text { comparação com uma criança saudável. } \\
\text { Recomendado a vacina após } 6 \text { meses sem a } \\
\text { quimioterapia. }\end{array}$ & Não \\
\hline 12 & Influenza & $\begin{array}{l}\text { Alcança o nível de corte para soroproteção. } \\
\text { Infelizmente, esse não é o caso em pacientes } \\
\text { tratados com rituximabe. }\end{array}$ & Sim \\
\hline 13 & Influenza & - & Sim \\
\hline 14 & Influenza & $\begin{array}{l}\text { Gera uma resposta imune contra a influenza (não } \\
\text { está claro se esta resposta imune os protege da } \\
\text { infecção por influenza ou de suas complicações) }\end{array}$ & Sim \\
\hline 15 & Influenza & $\begin{array}{l}\text { Estudos têm implicado protocolos baseados em } \\
\text { rituximab como associados a respostas vacinais } \\
\text { prejudicadas; caso contrário, protocolos } \\
\text { específicos não parecem afetar as respostas da } \\
\text { vacina. Pacientes com tumores sólidos, como foi } \\
\text { visto em crianças, parecem ter respostas } \\
\text { ligeiramente melhores do que aqueles com } \\
\text { neoplasias hematológicas }\end{array}$ & Sim \\
\hline 16 & Influenza & - & - \\
\hline 17 & Influenza & - & - \\
\hline 18 & Influenza & $\begin{array}{l}\text { Pacientes com tumores sólidos montaram } \\
\text { resposta imunológica(soroproteção) semelhante } \\
\text { ao indivíduo saudável. A melhor proteção é } \\
\text { identificada a vacinação antes do início da } \\
\text { quimioterapia. }\end{array}$ & Sim \\
\hline 19 & Varicela & $\begin{array}{l}\text { Em paciente oncológico pediátrico IgG negativo } \\
\text { a imunidade adaptativa foi induzida apesar da } \\
\text { soroconversão incompleta. (vacinar na fase } \\
\text { inicial do tratamento). A soroconversão foi de } \\
70 \% \text { após } 2 \text { vacinações. }\end{array}$ & Sim \\
\hline 20 & Influenza & $\begin{array}{l}\text { A vacina trivalente com alta dose de influenza } \\
\text { pode ser administrada com segurança a pacientes } \\
\text { que recebem quimioterapia com } \\
\text { imunogenicidade e soroconversão melhoradas }\end{array}$ & Sim \\
\hline
\end{tabular}




\begin{tabular}{|c|c|c|c|}
\hline & & em comparação com a vacina de dose padrão & \\
\hline 21 & Hepatite B & $\begin{array}{l}\text { Apresentou alta taxa de soroprevalência em } \\
\text { crianças com neoplasias malignas no Sudão. }\end{array}$ & Sim \\
\hline 22 & Influenza trivalente & $\begin{array}{l}\text { As taxas de soroproteção e soroconversão foram } \\
55 \text { e } 43 \% \text { para o H3N2, } 61 \text { e } 43 \% \text { para o H1N1, e } \\
41 \text { e 33\% para a cepa B, respectivamente. A } \\
\text { soroconversão foi significativamente mais } \\
\text { provável em crianças com doença sólida em } \\
\text { comparação com neoplasias hematológicas }\end{array}$ & Sim \\
\hline 23 & Influenza & - & - \\
\hline \multirow{3}{*}{24} & $\begin{array}{l}\text { DTaP, hepatite B, conjugado } \\
\text { meningocócico (MenC-C ou MenC- } \\
\text { ACWY) e varicela. }\end{array}$ & $\begin{array}{l}\text { Reimunização após a quimioterapia (Para } \\
\text { pacientes com leucemia - lla) }\end{array}$ & - \\
\hline & $\begin{array}{l}\text { Influenza trivalente inativada e vacina } \\
\text { meningocócica conjugada (MenC } \\
\text { sorogrupo C e MenC-ACWY) }\end{array}$ & $\begin{array}{l}\text { Recomendada durante a quimioterapia de } \\
\text { manutenção (Para pacientes com leucemia-1la). }\end{array}$ & - \\
\hline & Influenza & Única vacina recomendada fase intensiva da QT & Sim \\
\hline
\end{tabular}

Fonte: Autores.

O Quadro 3 destaca a ocorrência de 11 vacinas diferentes, repetidas nos estudos, exceto as vacinas contra rubéola e coqueluche. Destas 11 vacinas, estão descritas aquelas que foram testadas em pacientes oncológicos e também aquelas que foram estudadas por diferentes órgãos e associações.

Em um universo de 24 artigos, 83,3\% destes incluíram a vacina contra influenza, este que foi um dos destaque da análise, por ser uma vacina com maior taxa de soroconversão para pacientes oncológicos sem que haja a interrupção da quimioterapia e por ser indicada pelos profissionais de saúde, promovendo o cuidado e prevenção de doenças que possam diminuir ou anular a efetividade de cura do câncer.

Vale destacar que a maior parte dos estudos revisados foi com testes de vacinas em pacientes na fase inicial do tratamento, e para as vacinas indicadas para este grupo, é importante que haja um acompanhamento da equipe de assistência ao paciente oncológico.

Sabe- se que as vacinas de vírus vivos são contraindicadas em pacientes imunodeprimidos, no entanto, foi identificado em um artigo a soroconversão da vacina de vírus vivo contra varicela em pacientes oncológicos, sem interrupção da quimioterapia. Outro ponto de destaque são os que artigos deixaram em aberto esta questão para outras pesquisas. Três artigos relataram que tumores sólidos apresentam melhor soroconversão quando comparado ao câncer hematológico.

Ressalta-se também a identificação nos estudos revisados que as vacinas contra influenza, são efetivas na ação de imunoprevenção dos indivíduos que se encontram em tratamento, com destaques para hepatite B e varicela, esta última não protocolada nas orientações que regulamentam as atividades nos serviços de saúde da atenção primária em saúde.

Em relação à análise de soroconversão houve artigos que não indicaram a taxa de soroconversão, com o percentual de 37,5 \% dos artigos, com indicação da cobertura vacinal em pacientes oncológicos com vacinas que poderiam ser administradas. Dois artigos apresentaram a ineficácia da imunização para pacientes que fazem uso do quimioterápico rituximabe, nos quais uma resposta imune fica debilitada por esta associado a depleção persistente de células B de memória, como foi o caso da vacina 
influenza. A resposta positiva para soroconversão foi apresentada 15 vezes, com a resposta "SIM" no quadro 3, 13 para a influenza, 1 para varicela e 1 para hepatite $\mathrm{B}$.

A análise mostrou que a administração de algumas vacinas é indicada após o término do tratamento quimioterápico, o que gera boa taxa de soroconversão e não prejudica a saúde do paciente. O distanciamento para as vacinas são de 03 a 12 meses, depois de interrompido a quimioterapia. As vacinas são: DT, poliomielite, caxumba, Sarampo, rubéola, pneumocócica conjugada, Varicela (VZV), coqueluche, hepatite B conjugado meningocócico (MenC-C ou MenC-ACWY).

Para os pacientes tratados com leucemia linfoblástica aguda, LLA, as vacinas destacadas nos estudos foram: Influenza trivalente inativada e vacina meningocócica conjugada (MenC sorogrupo C e MenC-ACWY) com recomendação durante o tratamento de manutenção, mas abre espaço para maiores estudos que levaram a efetividade ou não desta vacinação.

\section{Considerações Finais}

A enfermagem tem um papel importante na imunização, participando no processo de educação e informação em saúde, mas também como cuidado em imunização, e este estudo denota que o pouco interesse pelo tema, ou o não reconhecimento da importância da vacinação no paciente oncológico como objeto de trabalho da enfermagem proporciona publicação científica da prática desta área por outras afins. Durante a análise identificou-se que apenas um artigo publicado por uma revista da área.

$\mathrm{Na}$ ótica da qualidade definida pela CAPES de produções da área da Enfermagem, os produtos, em sua maioria, não foram classificados, e ao se relacionar com a especialidade das revistas, a Enfermagem aparece 01 vez na publicação da Revista Clinical Journal of Oncology Nursing. Entender onde estão estas publicações e seus resultados, demonstram, mais uma vez, o papel do enfermeiro sendo conduzido pela produção do conhecimento realizado por outras áreas ${ }^{10}$

Além disso, os estudos mostraram intervenções em imunização que estão em voga no cuidado ao paciente neoplásico em tratamento quimioterápico, tais como a vacinação da influenza. Por outro lado, vacinas que não estão estabelecidas em orientações ministeriais, como a varicela, tríplice viral, foram testadas como meio de vacinação anterior ao tratamento, que demonstrou perda de efetividade na soroconversão. Já esta vacinação na fase inicial da quimioterapia, a resposta chegou a níveis de $70 \%$ de soroconversão.

Conclui-se, então, que este estudo é de importante repercussão, pois traça em uma única pesquisa o perfil das publicações sobre os pontos de interface de imunização em pacientes neoplásicos em tratamento quimioterápico nas plataformas de buscas de publicações científicas, mas também alerta sobre a prática da enfermagem em imunização como processo de construção do conhecimento por outras áreas, com reflexos na condução profissional. E por fim, demarca-se a necessidade novos estudos sobre a temática, especialmente oriundos da Enfermagem, com testes e análises de dados colhidos nos campos de atuação profissional.

\section{Referências}

Abdalla, M., \& Hamad, T. (2016). Hepatitis B virus Seroprevalence among children with cancer in Sudan. Pediatric Blood \& Cancer, 63(1), 124-126. https://doi.org/10.1002/pbc.25720

Ariza-Heredia, E. J., Azzi, J., Shah, D. P., Nesher, L., Ghantoji, S. S., Michailidis, L., Marsh, L., \& Chemaly, R. F. (2015). Influenza vaccination in patients with cancer: factors associated with vaccination practices for patients and their household members. Infect Control Hosp Epidemiol, 36(10), 1239-1241. https://doi.org/10.1017/ice.2015.150

Cesaro, S., Giacchino, M., Fioredda, F., Barone, A., Battisti, L., Bezzio, S., Frenos, S., Santis, R., Livadiotti, S., Marinello, S., Zanazzo, A. G., Caselli, D. (2014). Guidelines on vaccinations in paediatric haematology and oncology patients. BioMed Research International, 2014. https://doi.org/10.1155/2014/707691

Choi, K. H., Park, S. M., Lee, K., Lee, J. H., \& Park, J. S. (2014). Influenza vaccination and associated factors among Korean cancer survivors: a cross-sectional analysis of the Fourth \& Fifth Korea National Health and Nutrition Examination Surveys. Journal Of Korean Medical Science, 29(8), 1061-1068. https://doi.org/10.3346/jkms.2014.29.8.1061 
Eliakim-Raz, N., Vinograd, I., Trestioreanu, A. Z., Leibovici, L., \& Paul, M. (2013). Influenza vaccines in immunosuppressed adults with cancer. Cochrane Database of Systematic Reviews, (10). https://doi.org/10.1002/14651858.CD008983.pub3

Freedman, J. L., Reilly, A. F., Powell, S. C., \& Bailey, L. C. (2015). Quality improvement initiative to increase influenza vaccination in pediatric cancer patients. Pediatrics, 135(2), e540-e546. https://doi.org/10.1542/peds.2014-0943

Garcia, I. F., Coïc, L., Leclerc, J. M., Laverdière, C., Rousseau, C., Ovetchkine, P., \& Tapiéro, B. (2017). Protection against vaccine preventable diseases in children treated for acute lymphoblastic leukemia. Pediatric Blood \& Cancer, 64(2), 315-320. https://doi.org/10.1002/pbc.26187

Goossen, G. M., Kremer, L. C., \& Van De Wetering, M. D. (2013). Influenza vaccination in children being treated with chemotherapy for cancer. Cochrane Database of Systematic Reviews, (8). https://doi.org/10.1002/14651858.CD006484.pub3

Hakim, H., Allison, K. J., Van de Velde, L. A., Tang, L., Sun, Y., Flynn, P. M., \& McCullers, J. A. (2016). Immunogenicity and safety of high-dose trivalent inactivated influenza vaccine compared to standard-dose vaccine in children and young adults with cancer or HIV infection. Vaccine, 34(27), 3141-3148. https://doi.org/10.1016/j.vaccine.2016.04.053

Hanitsch, L. G., Löbel, M., Mieves, J. F., Bauer, S., Babel, N., Schweiger, B., \& Scheibenbogen, C. (2016). Cellular and humoral influenza-specific immune response upon vaccination in patients with common variable immunodeficiency and unclassified antibody deficiency. Vaccine, 34(21), 2417-2423. https://doi.org/10.1016/j.vaccine.2016.03.091

Jamshed, S., Walsh, E. E., Dimitroff, L. J., Santelli, J. S., \& Falsey, A. R. (2016). Improved immunogenicity of high-dose influenza vaccine compared to standard-dose influenza vaccine in adult oncology patients younger than 65 years receiving chemotherapy: a pilot randomized clinical trial. Vaccine, 34(5), 630635. https://doi.org/10.1016/j.vaccine.2015.12.037

Kelley, J., Tristram, D., Yamada, M., \& Grose, C. (2015). Failure of a single varicella vaccination to protect children with cancer from life-threatening breakthrough varicella. The Pediatric Infectious Disease Journal, 34(9), 1027. https://doi.org/10.1097/INF.0000000000000737

Kersun, L. S., Reilly, A. F., Coffin, S. E., \& Sullivan, K. E. (2013). Protecting pediatric oncology patients from influenza. The Oncologist, $18(2)$, 204. https://doi.org/10.1634/theoncologist.2012-0401

Kotecha, R. S., Wadia, U. D., Jacoby, P., Ryan, A. L., Blyth, C. C., Keil, A. D., Gottardo, N. G., Cole, C. H., Barr, I. G., \& Richmond, P. C. (2016). Immunogenicity and clinical effectiveness of the trivalent inactivated influenza vaccine in immunocompromised children undergoing treatment for cancer. Cancer Medicine, 5(2), 285-293. https://doi.org/10.1002/cam4.596

Lisy, K. (2014). Influenza vaccines in immunosuppressed adults with cancer. Clinical Journal of Oncology Nursing, 18(3). https://www.cochranelibrary.com/cdsr/doi/10.1002/14651858.CD008983.pub2/full

Ministério da Saúde. (2014). Manual do Centro de Referência para Imunobiológicos Especiais. https://portalarquivos2.saude.gov.br/images/pdf/2014/dezembro/09/manual-cries-9dez14-web.pdf

Oh, M. G., Han, M. A., Yun, N. R., Park, J., Ryu, S. Y., Kim, D. M., \& Choi, S. W. (2015). A population-based, nationwide cross-sectional study on influenza vaccination status among cancer survivors in Korea. International Journal of Environmental Research and Public Health, 12(8), 10133-10149. https://doi.org/10.3390/ijerph120810133

Ojha, R. P., Offutt-Powell, T. N., \& Gurney, J. G. (2014). Influenza vaccination coverage among adult survivors of pediatric cancer. American Journal Of Preventive Medicine, 46(6), 552-558. https://doi.org/10.1016/j.amepre.2014.01.007

Oliveira, V. G. D., Pedrosa, K. K. D. A., Monteiro, A. I., \& Santos, A. D. B. D. (2010). Vacinação: o fazer da enfermagem e o saber das mães e/ou cuidadores. http://www.repositorio.ufc.br/bitstream/riufc/13941/1/2010_art_vgoliveira.pdf

Pedrazzoli, P., Baldanti, F., Donatelli, I., Castrucci, M. R., Puglisi, F., Silvestris, N., \& Cinieri, S. (2014). Vaccination for seasonal influenza in patients with cancer: recommendations of the Italian Society of Medical Oncology (AIOM). Annals of oncology, 25(6), 1243-1247. https://doi.org/10.1093/annonc/mdu114

Queiroz, S. A., Moura, E. R. F., Nogueira, P. S. F., de Oliveira, N. C., \& Pereira, M. M. Q. (2009). Atuação da equipe de enfermagem na sala de vacinação e suas condições de funcionamento. Revista da Rede de Enfermagem do Nordeste, 10(4), 126-135. http://www.redalyc.org/articulo.oa?id=324027968015

Sanada, Y., Yakushijin, K., Nomura, T., Chayahara, N., Toyoda, M., Minami, Y., Matsuoka, H. (2016). A prospective study on the efficacy of two-dose influenza vaccinations in cancer patients receiving chemotherapy. Japanese Journal of Clinical Oncology, 46(5), 448-452. https://europepmc.org/article/med/26977053

Silva Junior, J. B. (2013). 40 years of the National Immunization Program: a conquest of Brazilian Public Health. http://dx.doi.org/10.5123/S167949742013000100001

Souza, M. T. D., Silva, M. D. D., \& Carvalho, R. D. (2010). Revisão integrativa: o que é e como fazer. Einstein (São Paulo), 8(1), 102-106. http://dx.doi.org/10.1590/s1679-45082010rw1134

Stafford, K. A., Sorkin, J. D., \& Steinberger, E. K. (2013). Influenza vaccination among cancer survivors: disparities in prevalence between blacks and whites. Journal of Cancer Survivorship, 7(2), 183-190. https://doi.org/10.1007/s11764-012-0257-3

Stillwell, S. B., Fineout-Overholt, E., Mazurek Melnyk, B., \& Williamson, K. M. (2010). Searching for the Evidence. The American Journal Of Nursing, 110(5), 41-47. http://www.nursingcenter.com/nursingcenter_redesign/media/EBP/AJNseries/Searching.pdf

Tavares, E. C., Ribeiro, J. G., \& Oliveira, L. A. (2005). Imunização ativa e passiva no prematuro extremo. J Pediatr (Rio J), 81(1 Supl), S89-94. https://www.scielo.br/pdf/jped/v81n1s1/v81n1s1a11.pdf 
Research, Society and Development, v. 10, n. 1, e10210111516, 2021

(CC BY 4.0) | ISSN 2525-3409 | DOI: http://dx.doi.org/10.33448/rsd-v10i1.11516

Toleman, M. S., Herbert, K., McCarthy, N., \& Church, D. N. (2016). Vaccination of chemotherapy patients-effect of guideline implementation. Supportive Care in Cancer, 24(5), 2317-2321. https://doi.org/10.1007/s00520-015-3037-6

Top, K. A., Pham-Huy, A., Price, V., Sung, L., Tran, D., Vaudry, W., Halperin, S. A., \& De Serres, G. (2016). Immunization practices in acute lymphocytic leukemia and post-hematopoietic stem cell transplant in Canadian Pediatric Hematology/Oncology centers. Human Vaccines \& Immunotherapeutics, 12(4), 931936. https://doi.org/10.1080/21645515.2015.1115165

Van de Wetering, M. D., Vossen, M. T., Jansen, M. H., Caron, H. N., Kuijpers, T. W. (2016). Varicella vaccination in pediatric oncology patients without interruption of chemotherapy. J Clin Virol. 75, 47-52. https://doi.org/10.1016/j.jcv.2016.01.004

Vinograd, I., Baslo, R., Eliakim-Raz, N., Farbman, L., Taha, A., Sakhnini, A., Lador, A., Stemmer, S.M., Gafter-Gvili, A., Fraser, D., Leibovici, L., Paul, M (2014). Factors associated with influenza vaccination among adult cancer patients: a case-control study. Clinical Microbiology and Infection, 20(9), 899-905. https://doi.org/10.1111/1469-0691.12625

Vollaard, A., Schreuder, I., Slok-Raijmakers, L., Opstelten, W., Rimmelzwaan, G., \& Gelderblom, H. (2017). Influenza vaccination in adult patients with solid tumours treated with chemotherapy. European Journal Of Cancer, 76, 134-143. https://doi.org/10.1016/j.ejca.2017.02.012 